\title{
Carbon monoxide releasing molecule-3 promotes the osteogenic differentiation of rat bone marrow mesenchymal stem cells by releasing carbon monoxide
}

\author{
JINGYUAN LI ${ }^{1}$, LING SONG ${ }^{2}$, MENG HOU $^{3}$, PING WANG $^{1}$, LINGLING WEI $^{1}$ and HUI SONG ${ }^{1}$ \\ ${ }^{1}$ School of Dentistry, Shandong Provincial Key Laboratory of Oral Tissue Regeneration, Shandong University, Jinan, \\ Shandong 250012; ${ }^{2}$ Department of Stomatology, Qingdao Municipal Hospital, Qingdao, Shandong 266011; \\ ${ }^{3}$ School of Stomatology, Medical College of Jining, Jining, Shandong 272000, P.R. China
}

Received August 6, 2017; Accepted January 9, 2018

DOI: $10.3892 / \mathrm{ijmm} .2018 .3437$

\begin{abstract}
Stem cell-based therapies are promising strategies to stimulate bone regeneration. Carbon monoxide releasing molecule-3 (CORM-3) exhibits multiple regulatory effects in a number of cells by releasing carbon monoxide (CO). The present study aimed to investigate the influence of CORM- 3 on the osteogenic differentiation of rat bone marrow mesenchymal stem cells (BMSCs). BMSCs were divided into five groups: A CORM-3-osteogenic group, in which cells were pretreated with CORM-3 and subjected to osteogenic differentiation induction using osteogenic medium; an osteogenic group, in which cells were cultured in osteogenic medium; a degassed CORM-3-osteogenic group, in which cells were pretreated with degassed CORM-3 and subjected to osteogenic differentiation induction; a CORM-3 group, in which cells were cultured in control medium containing CORM-3; and a control group, in which cells were cultured in control medium alone. The osteo-specific mRNA and protein expression of runt-related transcription factor 2 (Runx2), osteocalcin (OCN) and osteopontin (OPN) were assessed using reverse transcription-quantitative polymerase chain reaction and western blot analysis. Alkaline phosphatase (ALP) activity was also examined and mineralization was detected using alizarin red staining. Levels of Runx2, OCN and OPN mRNA and protein in the CORM-3-osteogenic group were significantly increased compared with the osteogenic group $(\mathrm{P}<0.05)$, with the exception of OCN protein levels on day 3 . The mRNA and protein expression of Runx2, OCN and OPN in the degassed CORM-3-osteogenic and osteogenic groups were similar. In
\end{abstract}

Correspondence to: Professor Hui Song, School of Dentistry, Shandong University, 44-1 Wenhua Xilu, Jinan, Shandong 250012, P.R. China

E-mail: songhui@sdu.edu.cn

Key words: carbon monoxide releasing molecule-3, bone marrow mesenchymal stem cells, osteogenic differentiation, runt-related transcription factor 2, osteocalcin, osteopontin addition, the mRNA and protein expression of Runx2, OCN and OPN in the CORM-3 and control group were similar. ALP activity in the CORM-3-osteogenic group was increased from day 3 and remained significantly higher compared with all other groups on days 3,5 and $7(\mathrm{P}<0.05)$. Additionally, the results indicated that the optical density value of alizarin red staining in the CORM-3-osteogenic group was significantly increased compared with the other groups $(\mathrm{P}<0.05)$. Therefore, the present study demonstrated that CORM-3 may promote the osteogenic differentiation of BMSCs by releasing $\mathrm{CO}$.

\section{Introduction}

Bone defects are usually caused by severe trauma, tumor resection and periodontal disease $(1,2)$. The limited intrinsic regenerative ability of the bone following destruction remains a notable medical problem and is associated with a severe reduction in the quality of patients. Therefore, it is important to develop methods of stimulating bone regeneration.

Bone marrow mesenchymal stem cells (BMSCs) are stem cell populations capable of self-renewal and may be maintained in a multipotent state in vitro $(3,4)$. They are also one of the most important cells contributing to musculoskeletal tissue development (1). BMSCs differentiate into multiple lineages, including osteoblasts, chondrocytes, adipocytes and myoblasts via different signaling pathways $(5,6)$. Preserving bone morphology and function relies on maintaining the delicate balance between bone formation and resorption $(1,2)$. Osteoblasts, which form the bone, differentiate from BMSCs $(7,8)$. BMSCs are therefore currently regarded as the gold standard cell source for tissue engineering and applications in regenerative medicine (9). Previous studies have successfully demonstrated the potential of BMSCs in promoting bone regeneration using in vitro and in vivo models $(10,11)$.

Carbon monoxide (CO), a byproduct of heme catalysis by hemeoxygenase, has long been regarded as a poisonous gas. However, previous studies have indicated that CO may be cytoprotective as it induces vasorelaxation $(12,13)$, inhibits cell apoptosis (14), suppresses inflammation (15) and protects organs against ischemia/reperfusion injury $(16,17)$. CO 
releasing molecule (CORMs) are newly identified transition metal carbonyl-based compounds able to efficiently regulate the release of $\mathrm{CO}$ in vitro and in vivo under appropriate conditions (18). These molecules may therefore be used as a novel approach of delivering CO. One such CORM, known as CORM-3 [tricarbonylchloro (glycinato) ruthenium (II)], is fully water-soluble and has the ability to rapidly liberate $\mathrm{CO}$ when dissolved in physiological solutions (19). As with $\mathrm{CO}, \mathrm{CORMs}$ exhibit potent anti-inflammatory effects (20). Furthermore, CORMs are able to improve vascular function by inducing significant vasodilation in rat aortas pre-contracted with phenylephrine (19).

Although various studies (18-20) have suggested that CORMs induce beneficial effects, the effect of CORMs on osteogenic differentiation remains unclear. In the present study, rat BMSCs were used as an in vitro model to investigate the effect of CORM-3 on osteogenic differentiation.

\section{Materials and methods}

Isolation and culture of rat BMSCs. A total of 6 male Sprague Dawley rats (specific pathogen-free grade, aged 4-5 weeks old and weighing 100-150 g) were obtained from the Animal Experimental Center of Shandong University (Jinan, China). Rats were kept at a temperature of $20-25^{\circ} \mathrm{C}$, humidity of 50-65\% and 12-h light-dark cycle. Rats had ad libitum access to food and water. The present study was approved by the Ethics Committee of the School of Dentistry, Shandong University. Rats were euthanized by intraperitoneal injection of buffered and diluted pentobarbital sodium $(150 \mathrm{mg} / \mathrm{kg}$; Beijing Solarbio Science \& Technology Co., Ltd., Beijing, China), then soaked in $75 \%$ alcohol at $37^{\circ} \mathrm{C}$ for $10 \mathrm{~min}$. Subsequently, the femur and tibia were removed and the bone marrow cavity was rinsed with $\alpha$-Minimum Essential Medium (MEM) (Shanghai Hui Ying Biological technology Co., Ltd., Shanghai, China) supplemented with $20 \%$ fetal bovine serum (FBS) and $100 \mathrm{U} / \mathrm{ml}$ penicillin-streptomycin (HyClone; GE Healthcare Life Sciences, Logan, UT, USA). Bone marrow fluid was incubated at $37^{\circ} \mathrm{C}$ in an atmosphere containing $5 \% \mathrm{CO}_{2}$. The medium was changed every 2-3 days, non-adherent cells were discarded and cells were observed using the Olympus CKX53 phase-contrast microscope (Olympus Corporation, Tokyo, Japan). When cells reached 80-90\% confluence, BMSCs were digested with $0.25 \%$ Trypsin (HyClone; GE Healthcare Life Sciences) containing $0.02 \%$ EDTA. BMSCs from passages (P) 3 to 5 were used in the present study.

Flow cytometric identification of BMSC surface antigens. BMSCs were digested with $0.25 \%$ Trypsin containing $0.02 \%$ EDTA (HyClone; GE Healthcare Life Sciences,), rinsed with phosphate buffered saline (PBS; Beijing Solarbio Science \& Technology Co., Ltd.) three times, then resuspended in $0.5 \mathrm{ml}$ PBS. Cell density was $2 \times 10^{5} / \mathrm{ml}$, assessed using a cell counting plate (Shanghai QIUJING Biochemical Reagent and Instrument Co., Ltd., Shanghai, China). Anti-cluster of differentiation (CD)45-fluorescein isothiocyanate (FITC; cat no., ab33916, dilution 1:500), anti-CD34-phycoerythrin (PE; cat. no., ab187284, dilution 1:1000), anti-CD90-FITC (cat. no., ab226, dilution 1:500) and anti-CD44-PE (cat. no., ab23396, dilution 1:500) monoclonal antibodies (all from Abcam, Cambridge, MA, USA) were added separately and were incubated with cells in the dark at $37^{\circ} \mathrm{C}$ for $20 \mathrm{~min}$. Cells were then rinsed with PBS three times, centrifuged at $4^{\circ} \mathrm{C}$ and $12,000 \times \mathrm{g}$ for $5 \mathrm{~min}$ and resuspended in PBS. Subsequently, labeled BMSCs were analyzed using a FACSCalibur flow cytometer and assessed using FACSDiva ${ }^{\mathrm{TM}}$ Version 6.1.3 (BD Biosciences, Franklin Lakes, NJ, USA). Unstained cells were used as a control.

Alizarin red staining. P3 BMSCs were seeded in 6-well plates at density of $8 \times 10^{3}$ cells $/ \mathrm{cm}^{2}$ and cultured in osteogenic medium, consisting of $\alpha$-MEM medium supplemented with $50 \mu \mathrm{mol} / 1$ ascorbic acid (Sigma-Aldrich; Merck KGaA, Darmstadt, Germany), $10 \mathrm{mmol} / 1 \beta$-glycerophosphate (Sigma-Aldrich; Merck KGaA) and $10^{-8} \mathrm{~mol} / 1$ dexamethasone (Beijing Solarbio Science \& Technology Co., Ltd.) at $37^{\circ} \mathrm{C}$. Cells in the control group were treated with $\alpha$-MEM supplemented with $10 \%$ FBS and $100 \mathrm{U} / \mathrm{ml}$ penicillin-streptomycin (the control medium) and cultured at $37^{\circ} \mathrm{C}$. Ascorbic acid was freshly prepared prior to each medium change. Following 21 days culture, cells were fixed with $4 \%$ paraformaldehyde at $37^{\circ} \mathrm{C}$ for $30 \mathrm{~min}$. Following three washes with PBS, cells were incubated with $0.1 \%(\mathrm{pH} 4.2)$ Alizarin Red S (Beijing Solarbio Science \& Technology Co., Ltd.) at $37^{\circ} \mathrm{C}$ for $10 \mathrm{~min}$ and subsequently washed with PBS three times to remove any excess stain. Samples were observed using a phase-contrast microscope at a magnification of x100 to verify the presence of mineralized nodules.

Oil red $O$ staining. P3 BMSCs were seeded as aforementioned and cultured in adipogenic medium, consisting of $\alpha$-MEM supplemented with $0.1 \mathrm{mmol} / 1$ 3-isobutyl-1-methylxanthine (Sigma-Aldrich; Merck KGaA), $10 \mathrm{mg} / 1$ insulin, $0.1 \mathrm{mmol} / 1$ indometacin, $1 \mu \mathrm{mol} / 1$ dexamethasone (all Beijing Solarbio Science \& Technology Co., Ltd.) at $37^{\circ} \mathrm{C}$. Cells in the control group were cultured in control medium at $37^{\circ} \mathrm{C}$. Following 21 days culture, cells were fixed in $4 \%$ paraformaldehyde at $37^{\circ} \mathrm{C}$ for $30 \mathrm{~min}$. Following three washes with PBS, cells were incubated with Oil Red O (Beijing Solarbio Science \& Technology Co., Ltd.) at $37^{\circ} \mathrm{C}$ for $30 \mathrm{~min}$ and subsequently washed with PBS three times. Samples were observed using phase-contrast microscopy at a magnification of $\mathrm{x} 100$.

Cell proliferation assay. P3 BMSCs were seeded in 96-well plates at a density of $5 \times 10^{3}$ cells $/ \mathrm{cm}^{2}$ and cultured in control medium for $24 \mathrm{~h}$ at $37^{\circ} \mathrm{C}$. Subsequently the medium was removed and cells were cultured in fresh control medium containing 0 (CCK-8 control group), 100, 200,400 and $800 \mu \mathrm{M}$ CORM-3. CORM-3 was freshly prepared prior to the experiment by dissolving the compound in distilled water. Following 24 h, $10 \mu$ l Cell Counting Kit-8 (CCK-8; Dojindo Molecular Technologies, Inc., Beijing, China) was added to each well and cells were incubated for a further $2 \mathrm{~h}$ at $37^{\circ} \mathrm{C}$. Subsequently, absorbance at $450 \mathrm{~nm}$ [optical density (OD) ${ }_{450 \mathrm{~nm}}$ ] was measured using a SPECTROstar Nano ultraviolet spectrophotometer (Spectro Analytical Instruments GmbH, Kleve, Germany). The experiment was repeated in triplicate.

Effects of CORM-3 on osteogenic differentiation. Cells were divided into 5 groups. The CORM-3-osteogenic group 
received BMSCs that were pretreated with control medium supplemented with $200 \mu \mathrm{M}$ CORM-3 at $37^{\circ} \mathrm{C}$ for $24 \mathrm{~h}$. Subsequently, the medium was completely replaced with osteogenic medium for 3 days. The osteogenic group received BMSCs cultured in $37^{\circ} \mathrm{C}$ osteogenic medium for 3 days. The degassed CORM-3-osteogenic group received BMSCs that were pretreated with control medium supplemented with $200 \mu \mathrm{M}$ degassed CORM-3 at $37^{\circ} \mathrm{C}$ for $24 \mathrm{~h}$. Following this, the medium was replaced with $37^{\circ} \mathrm{C}$ osteogenic medium for 3 days. The CORM-3 group received BMSCs cultured in control medium supplemented with $200 \mu \mathrm{M}$ CORM-3 at $37^{\circ} \mathrm{C}$ for 3 days. The control group received BMSCs that were cultured in control medium alone at $37^{\circ} \mathrm{C}$ for 3 days. For the experiments performed for 5 and 7 days, the medium was changed every third day in all groups.

CORM-3 was freshly prepared as aforementioned. Degassed CORM-3 was produced by dissolving CORM-3 in distilled water and placing the solution in a vacuum device at $37^{\circ} \mathrm{C}$ for $24 \mathrm{~h}$ prior to the experiments. Degassed CORM-3 was used as a negative control to assess the direct involvement of CO in the pharmacological activity of CORM-3 (18). For osteogenic differentiation experiments, BMSCs were seeded in 6-well plates at a density of $5 \times 10^{4}$ cells/well and cultured in the indicated medium. The mRNA and protein expression of osteoblast marker genes, alkaline phosphatase (ALP) activity and matrix mineralization were subsequently analyzed.

Reverse transcription-quantitative polymerase chain reaction (RT-qPCR). Following 3, 5 and 7 days culture, total RNA was isolated using TRIzol reagent (Thermo Fisher Scientific, Inc., Waltham, MA, USA), according to the manufacturer's protocol. cDNA was synthesized from $1 \mu \mathrm{g}$ total RNA using a PrimeScript Reverse Transcriptase reagent kit (Takara Biotechnology Co., Ltd., Dalian, China), following the manufacturer's protocol. cDNAs were subjected to qPCR with SYBR Green (Takara Biotechnology Co., Ltd.) on a Light Cycler II Real-time PCR system (Roche Diagnostics, Basel, Switzerland) to detect mRNA levels of runt-related transcription factor 2 (Runx2), osteocalcin (OCN) and osteopontin (OPN). The qPCR thermocycling conditions were as follows: Initial denaturation at $95^{\circ} \mathrm{C}$ for $15 \mathrm{~min}$, followed by 40 cycles of denaturation at $95^{\circ} \mathrm{C}$ for $10 \mathrm{sec}$, annealing at $60^{\circ} \mathrm{C}$ for $20 \mathrm{sec}$ and extension at $72^{\circ} \mathrm{C}$ for $30 \mathrm{sec}$. $\beta$-actin served as the housekeeping gene for normalization. The primer sequences used were as follows: Runx2, forward 5'-CAGACACAATCC TCCCCACC-3', and reverse 5'-GCCAGAGGCAGAAGTCAG AG-3'; OCN, forward 5'-ATTGTGACGAGCTAGCGGAC-3' and reverse 5'-TCGAGTCCTGGAGAGTAGCC-3'; OPN, forward 5'-TCAAGGTCATCCCAGTTGCC-3' and reverse 5'-GACTCATGGCTGGTCTTCCC-3'; and $\beta$-actin, forward 5'-CTCTGTGTGGATTGGTGGCT-3' and reverse 5'-CGC AGCTCAGTAACAGTCCG-3'. Each sample was tested in triplicate and gene expression levels were assessed using the LightCycler 480 Software 1.5 (Roche Diagnostics). Results were quantified using the $2^{-\Delta \Delta \mathrm{Cq}}$ method (21).

Western blot analysis. Following 3, 5 and 7 days culture, cells were washed twice with precooled PBS and treated with radio immunoprecipitation assay lysis buffer (Beijing Solarbio Science \& Technology Co., Ltd.) containing
$1 \mathrm{mmol} / \mathrm{l}$ phenylmethylsulfonyl fluoride protease inhibitors. The protein concentration of each group was measured using a BCA protein assay kit (Wuhan Boster Biological Technology Co., Ltd., Wuhan, China), following the manufacturer's protocol. Protein samples (20 $\mu \mathrm{g} / \mathrm{lane})$ were resolved using 12\% SDS-PAGE (Wuhan Boster Biological Technology Co., Ltd.) and electrotransferred to a polyvinylidene difluoride membrane (Pall Corporation, Port Washington, NY, USA). Following blocking in 5\% skimmed milk in Tris-buffered saline with $0.1 \%$ Tween-20 (TBST) at $37^{\circ} \mathrm{C}$ for $1 \mathrm{~h}$, the membrane was incubated overnight at $4^{\circ} \mathrm{C}$ with primary antibodies, including rabbit anti-rat Runx2 (cat no., 12556s, 1:1,000 dilution; Cell Signaling Technology, Inc., Danvers, MA, USA), mouse anti-rat OCN (cat no., ab13420, 1:500 dilution) and rabbit anti-rat OPN (cat no., ab8448, 1:1,000 dilution; both from Abcam). Following three washes with TBST, membranes were incubated with horseradish peroxidase-conjugated goat anti-rabbit or goat anti-mouse secondary antibodies (cat. nos., SA00001-2, SA00011; both 1:10,000 dilution, Proteintech Group, Inc., Rosemont, IL, USA) for $1 \mathrm{~h}$ at room temperature and washed three times with TBST. Following this, membranes were visualized using enhanced chemiluminescence (EMD Millipore, Billerica, MA, USA) and a ChemiScope Western Blot Imaging System (Clinx Science Instruments Co., Ltd., Shanghai, China). Loading differences were normalized using a monoclonal GAPDH antibody (cat. no., CW0100, 1:2,000 dilution; Beijing ComWin Biotech Co., Ltd., Beijing, China). Protein band densities on scanned films were quantified using ImageJ $1.48 \mathrm{u}$ software (National Institutes of Health, Bethesda, MD, USA) and compared with the control.

ALP activity assay. Following 3, 5 and 7 days culture, cells were washed twice with ice-cold PBS, harvested in $1 \%$ Triton and subsequently centrifuged at $4^{\circ} \mathrm{C}$ for $15 \mathrm{~min}$ at $12,000 \mathrm{x}$ g. The supernatant was mixed with an ALP kit (Nanjing Jiancheng Bioengineering Institute, Nanjing, China), following the manufacturer's protocol. OD values were read at $520 \mathrm{~nm}$ using an ELISA plate reader. The protein concentration of each sample was measured using a BCA protein assay kit. ALP activity was calculated according to the ALP kit protocol and expressed as King-Armstrong units/g of total cellular protein.

Analysis of mineralization. Following 21 days culture, mineralization was detected in all groups using alizarin red staining, as aforementioned. Staining was dissolved in $100 \mu \mathrm{M}$ cetylpyridinium chloride (CPC) for $1 \mathrm{~h}$ at $37^{\circ} \mathrm{C}$. For further evaluation, the $\mathrm{OD}$ value of the staining dissolved by $\mathrm{CPC}$ was measured at $562 \mathrm{~nm}$ using an ELISA plate reader. All experiments were repeated in triplicate.

Statistical analysis. Data were obtained from at least three independent experiments. The significance of differences was assessed by one-way analysis of variance method using GraphPad Prism 5 software (GraphPad Software, Inc., La Jolla, CA, USA), followed by the Tukey's post hoc test. Data are presented as the mean \pm standard deviation and $\mathrm{P}<0.05$ was considered to indicate a statistically significant difference. 

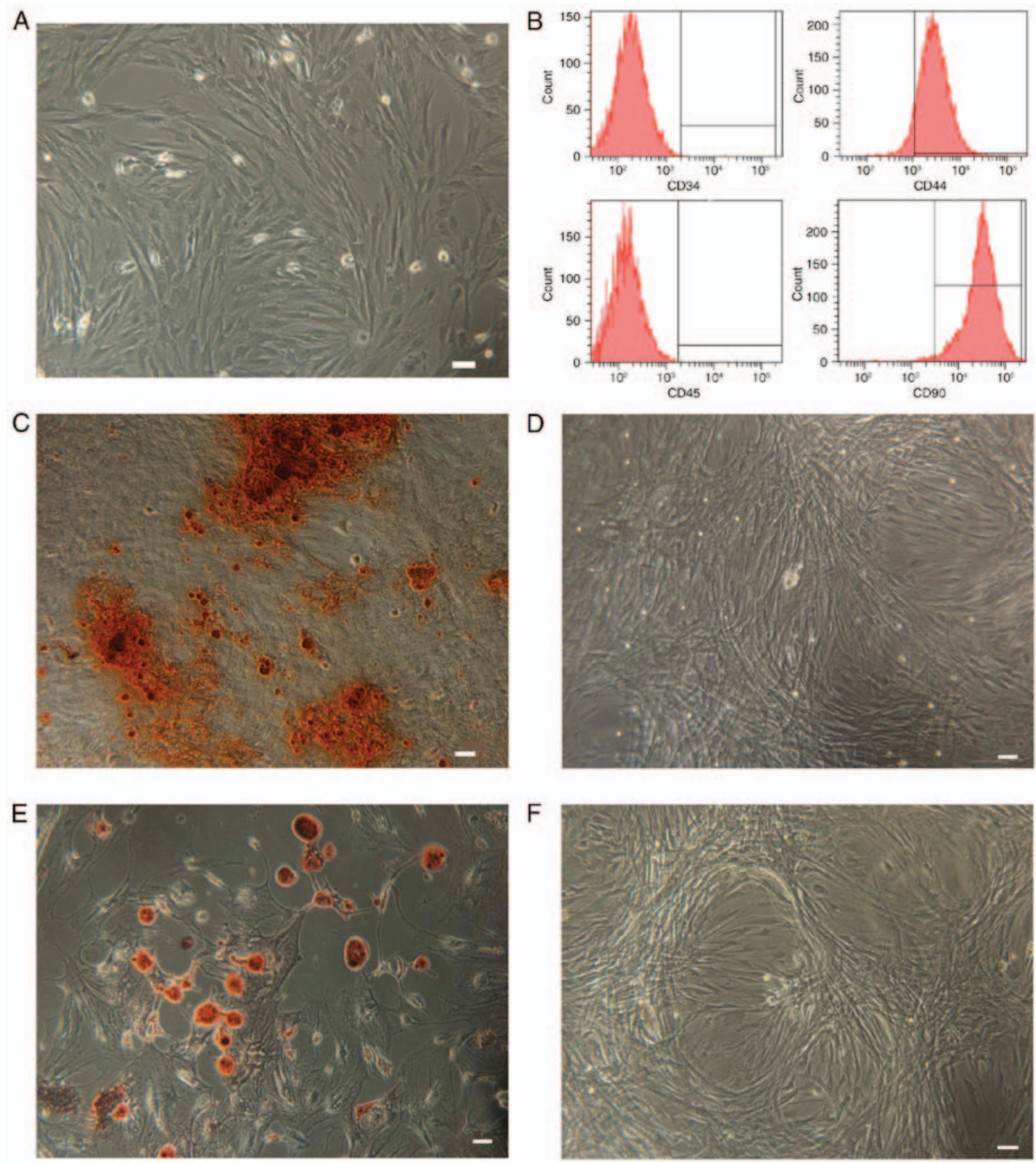

Figure 1. Identification of BMSCs. (A) BMSC morphology was assessed using a microscope. (B) Flow cytometry was performed to identify BMSC surface antigens. (C and D) Identification of osteogenic differentiation. Alizarin red staining of BMSCs cultured in the (C) osteogenic or (D) control medium for 21 days. (E and F) Adipogenic differentiation identification. Oil red $\mathrm{O}$ staining of BMSCs cultured in the (E) adipogenic or (F) control medium for 21 days. Magnification, x100; scale bar, $50 \mu \mathrm{m}$. BMSCs, bone marrow mesenchymal stem cells.

\section{Results}

Identification of BMSCs. As indicated in Fig. 1, cultured BMSCs were identified by assessing their morphology using different techniques. BMSCs presented with fibroblast-like morphological features (Fig. 1A). Flow cytometric analysis demonstrated that expression of the MSC surface markers CD90 (98.4\%) and CD44 (92.1\%) was high in BMSCs, whereas expression of the hematopoietic surface markers CD45 (0.2\%) and CD34 (0.1\%) was low (Fig. 1B). Furthermore, the multilineage differentiation potential of BMSCs was evaluated. BMSCs were induced to differentiate into osteoblasts or adipocytes. The mineralized matrix was visualized using alizarin red staining (Fig. 1C) and lipid droplets were identified using oil red $\mathrm{O}$ staining (Fig. 1E). Control cell cultures were negative for alizarin red (Fig. 1D) and oil red $\mathrm{O}$ staining (Fig. 1F).

Effect of CORM-3 on BMSC proliferation. BMSCs were cultured with different concentrations of CORM-3. The proliferative ability of the cells was assessed using a CCK-8 kit. As indicated in Fig. 2, 100 and $200 \mu \mathrm{M}$ CORM-3 significantly promoted the proliferation of BMSCs, whereas $400 \mu \mathrm{M}$ CORM-3 had no significant effect on cell proliferation compared with the control $(\mathrm{P}<0.05)$. Notably, $800 \mu \mathrm{M}$ 


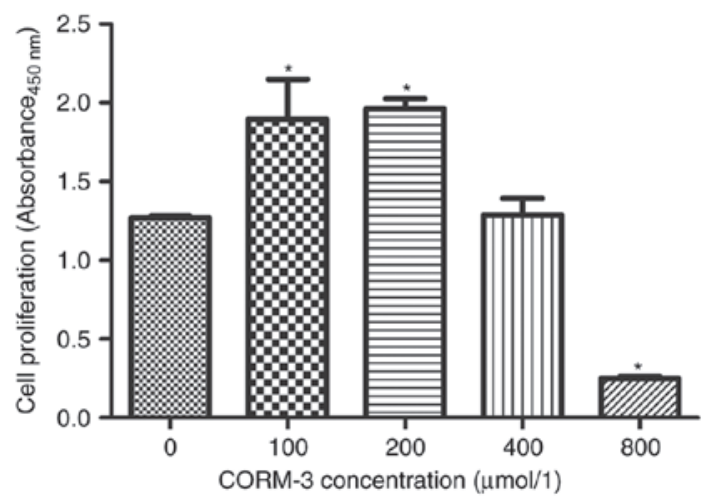

Figure 2. Effects of CORM-3 on bone marrow mesenchymal stem cell proliferation. Data are presented as the mean \pm standard deviation $(n=3)$. ${ }^{*} \mathrm{P}<0.05$ vs. control group. CORM-3, carbon monoxide releasing molecule-3.

CORM-3 significantly inhibited cell proliferation compared with the control $(\mathrm{P}<0.05)$. Based on these results, $200 \mu \mathrm{M}$ CORM-3 was selected for the following experiments.

Effect of CORM-3 on ALP activity during osteogenic differentiation. ALP activity was assessed on days 3, 5 and 7 (Fig. 3). ALP activity in the CORM-3-osteogenic group increased rapidly from day 3 and peaked on day 7; it was significantly higher compared with the other groups on days 3,5 and $7(\mathrm{P}<0.05)$. ALP activity was only significantly increased in the osteogenic group on day 7 compared with the control group $(\mathrm{P}<0.05)$. Notably, there were no significant differences in ALP activity in the CORM-3 group compared with the control group. The ALP activity in the degassed CORM-3-osteogenic group was not increased compared with the osteogenic group on days 3,5 and 7, suggesting that the influence of CORM-3 on ALP activity was mediated by $\mathrm{CO}$ release (Fig. 3).
Effects of CORM-3 on Runx2, OCN and OPN mRNA levels during osteogenic differentiation. To determine whether CORM-3 promotes the expression of osteo-specific genes, BMSCs were subjected to osteogenic differentiation with or without CORM-3 pretreatment. mRNA levels of the osteoblast key transcription factor Runx 2 and the osteoblast marker genes $\mathrm{OCN}$ and OPN, were assessed using RT-qPCR at different time points during osteogenic differentiation. Degassed CORM-3 was used to determine the involvement of $\mathrm{CO}$ on the effect of CORM-3. As indicated in Fig. 4, levels of Runx2 mRNA in cells cultured in osteogenic medium and pretreated with CORM-3 were significantly increased compared with all other groups on days 3,5 and $7(\mathrm{P}<0.05)$. By day 7, CORM-3 pretreatment in the CORM-3-osteogenic group had increased the expression of Runx 2 mRNA by 4.9 -fold compared with the control group $(\mathrm{P}<0.05)$, and 2.2 -fold compared with the osteogenic group $(\mathrm{P}<0.05)$. CORM-3 without osteogenic solution had no significant effect on the expression of Runx 2 mRNA compared with the control group. These results in CORM-3-osteogenic, osteogenic and degassed CORM-3-osteogenic groups suggest that the effect of CORM-3 on the expression of Runx2 mRNA was mediated by the release of $\mathrm{CO}$, as degassed CORM-3 was ineffective compared with osteogenic group. Similar effects were also observed regarding the expression of OPN mRNA. Levels of OCN mRNA peaked on day 5 in the CORM-3-osteogenic group and were significantly higher in the CORM-3-osteogenic group compared with all other groups at each time point $(\mathrm{P}<0.05)$.

Effects of CORM-3 on Runx2, OCN and OPN protein expression during osteogenic differentiation. The protein expression of Runx2 and OPN in the CORM-3-osteogenic group was significantly increased compared with all other groups at all time points $(\mathrm{P}<0.05$; Fig. 5$)$. The protein expression of Runx 2 in the CORM-3-osteogenic group was increased by 2.5 -fold compared with the control group,

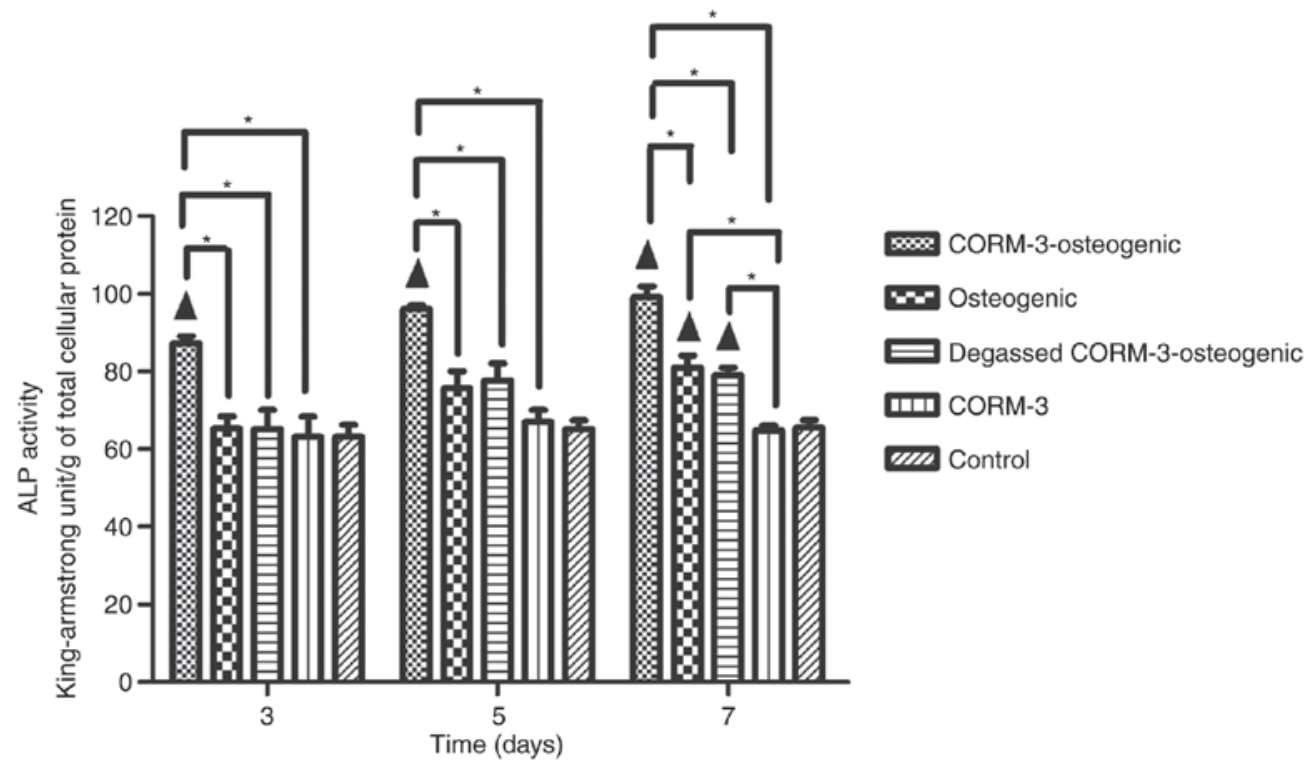

Figure 3. Quantitative evaluation of the osteogenic differentiation capacity of bone marrow mesenchymal stem cells using the ALP activity assay. Data are presented as the mean \pm standard deviation $(\mathrm{n}=3)$. ${ }^{\wedge} \mathrm{P}<0.05$ vs. Control; ${ }^{*} \mathrm{P}<0.05$ as indicated. ALP, alkaline phosphatase; CORM-3, carbon monoxide releasing molecule-3. 

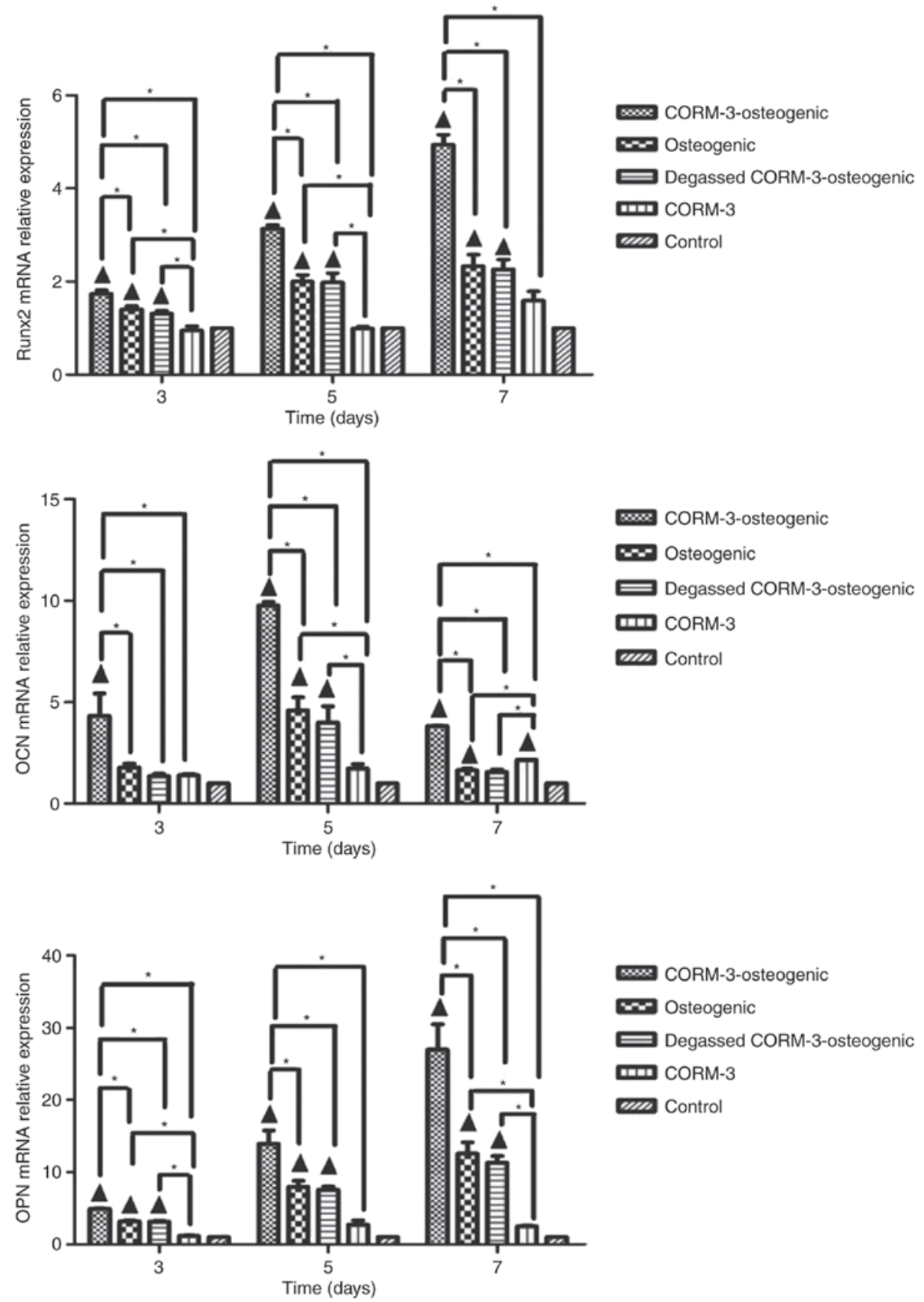

Figure 4. mRNA expression of the osteogenic differentiation markers Runx2, OCN and OPN in bone marrow mesenchymal stem cells following treatment with different mediums on 3, 5 and 7 days. Data are presented as the mean \pm standard deviation $(\mathrm{n}=3) .{ }^{\Delta} \mathrm{P}<0.05$ vs. Control; ${ }^{*} \mathrm{P}<0.05$ as indicated. Runx2, runt-related transcription factor 2; OCN, osteocalcin; OPN, osteopontin; CORM-3, carbon monoxide releasing molecule-3.

2.08-fold compared with the osteogenic group on day 5 , and 4.1-fold compared with the control group and 1.52-fold compared with osteogenic group on day $7(\mathrm{P}<0.05)$. The expression of OCN in the CORM-3-osteogenic group peaked on day 5 and then declined on day 7 . However, OCN expression remained significantly increased in the CORM-3-osteogenic group compared with all other groups on days 5 and $7(\mathrm{P}<0.05)$. CORM-3 alone had no significant effect on the protein expressions of Runx2, OCN and OPN compared with the control group. These results from the CORM-3-osteogenic, osteogenic and degassed CORM-3-osteogenic groups suggest that the influence of
CORM-3 on the protein expression of these factors was mediated by CO release, as the degassed solution of CORM-3 did not significantly increase the expression of any proteins tested, compared with osteogenic group (Fig. 5).

Effects of CORM-3 on mineralization. The results from the alizarin red staining demonstrated that mineralization in the CORM-3-osteogenic group was markedly enhanced compared with the osteogenic group after 21 days culture (Fig. 6A). To evaluate the mineralization in a quantitative manner, the $\mathrm{OD}$ value of the staining dissolved by $\mathrm{CPC}$ was analyzed. As indicated in Fig. 6B, alizarin red staining in the CORM-3-osteogenic 
A

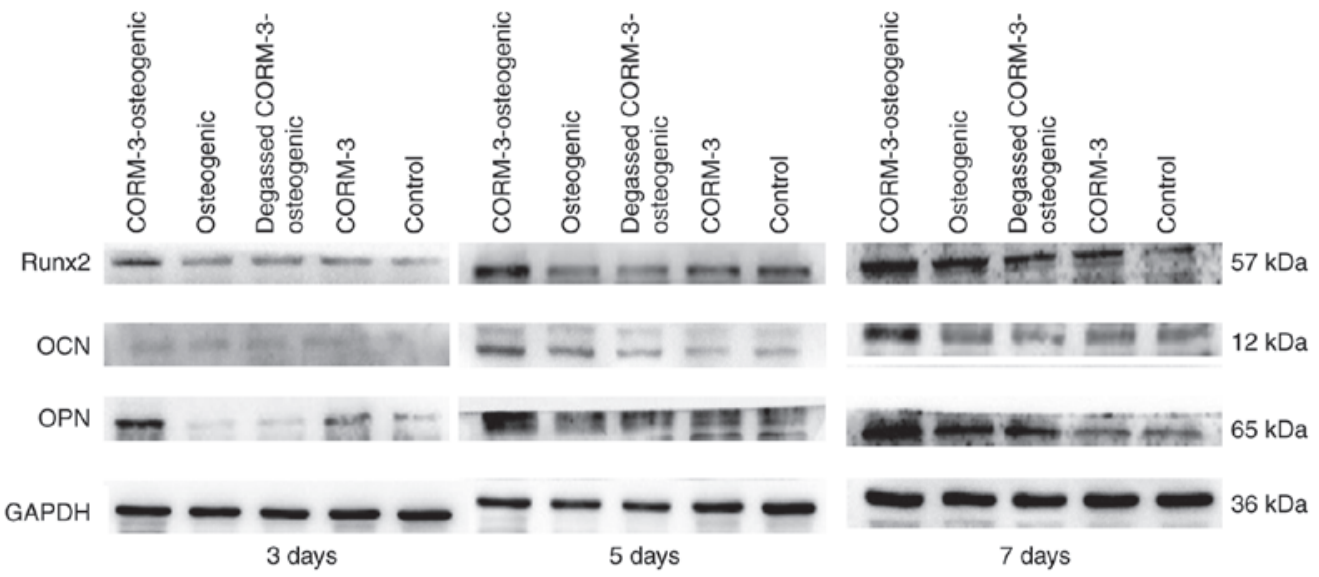

B
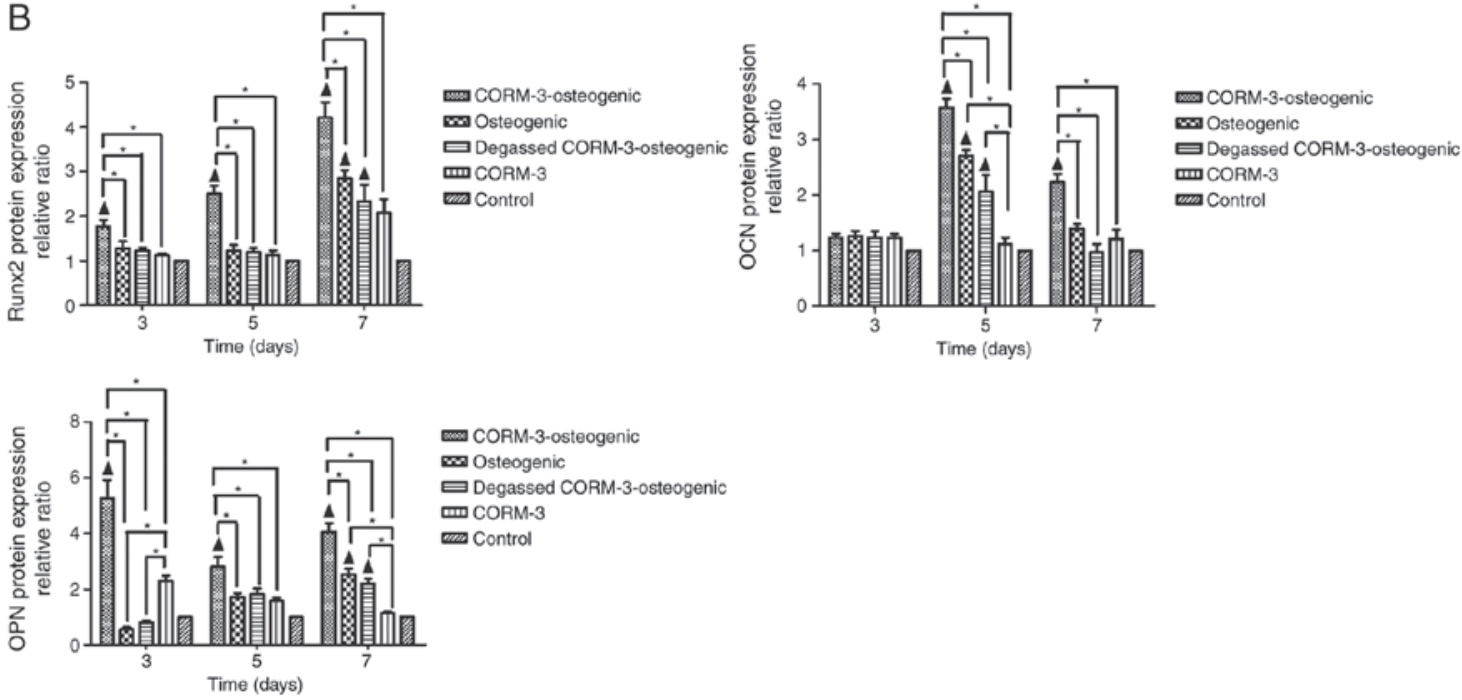

Figure 5. Protein expression of the osteogenic differentiation markers Runx2, OCN and OPN in bone marrow mesenchymal stem cells following treatment with different mediums on 3,5 and 7 days. (A) Representative western blot images of three independent experiments in all groups. (B) Quantitative results of western blot images in all groups using ImageJ software. Data are presented as the mean \pm standard deviation $(\mathrm{n}=3) .{ }^{\mathbf{\Delta}} \mathrm{P}<0.05$ vs. Control; ${ }^{*} \mathrm{P}<0.05$ as indicated. Runx2, runt-related transcription factor 2; OCN, osteocalcin; OPN, osteopontin; CORM-3, carbon monoxide releasing molecule-3.
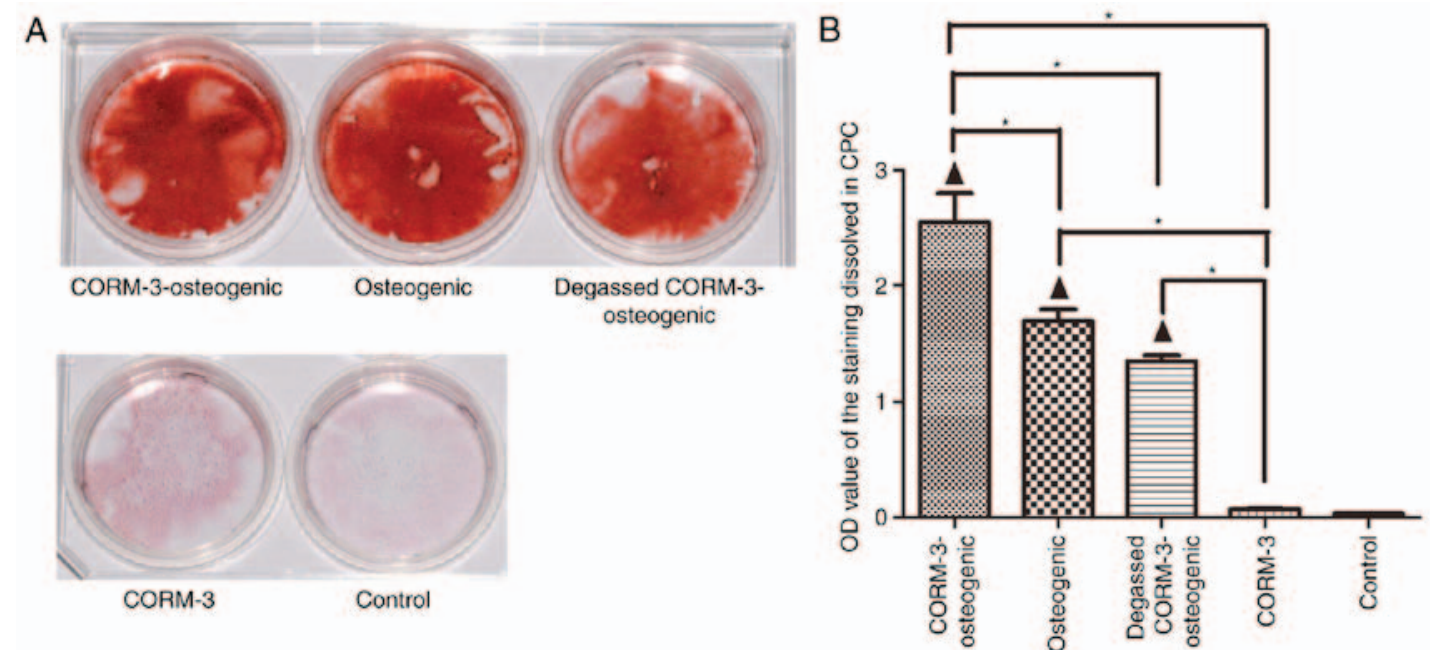

Figure 6. Effects of CORM-3 on mineralization during osteogenic differentiation of bone marrow mesenchymal stem cells. (A) Representative alizarin red staining images of all groups. (B) Quantitative results of alizarin red staining in all groups. Data are presented as the mean \pm standard deviation $\left(\mathrm{n}=3\right.$ ). ${ }^{\mathbf{\Delta}} \mathrm{P}<0.05$ vs. Control; " $\mathrm{P}<0.05$ as indicated. CORM-3, carbon monoxide releasing molecule-3; OD, optical density; CPC, cetylpyridinium chloride.

group was significantly higher than in the osteogenic groups $(\mathrm{P}<0.05)$. The osteogenic promotion effect of CORM-3 was mediated by releasing $\mathrm{CO}$ as the degassed CORM-3 did not affect osteogenesis compared with osteogenic group. 


\section{Discussion}

Previous studies have identified the beneficial effects of $\mathrm{CO}$ and CORMs; however, little is known regarding the influence of CORMs on osteogenesis. In the present study, rat BMSCs were used as an in vitro model to investigate the effects of CORM-3 on the osteogenic differentiation of BMSCs. The results suggested that 100 and $200 \mu \mathrm{M}$ CORM-3 significantly promotes the proliferation of BMSCs. Additionally, pretreatment with CORM-3 upregulated the mRNA and protein expression of the osteo-specific markers Runx 2, OCN and OPN. Notably, CORM-3 pretreatment also increased ALP activity and enhanced cell mineralization. The results of the present study suggest that these effects were mediated by the release of $\mathrm{CO}$.

Various diseases, including cancer, infection and degenerative diseases, may cause bone defects $(1,2)$. Stem cell-based bone engineering has emerged as a promising and effective alternative approach of treating these diseases (22). BMSCs are presently regarded as the gold standard cell source for bone tissue engineering, due to their great potential for self-renewal and their ability to differentiate into cell lineages characteristic of bone (23). The predominant objective of bone tissue engineering is to stimulate effective and sufficient bone regeneration, which is typically based on a successful osteoinduction. Multiple strategies have been implemented for osteoinduction, including the application of selected growth factors (24). Cytokines that promote osteogenesis include bone morphogenetic proteins (BMPs), platelet-derived growth factor and fibroblast growth factor (25). The osteogenic properties of BMPs have been determined in a number of studies, and recombinant human (rh)BMP2 and rhBMP7 have been approved by the US Food and Drug Administration for specific clinical applications $(26,27)$. However, recent investigations have reported notable side effects associated with rhBMP-2, including renal complications, wound complications, increased inflammation and an increased risk of cancer (25-29). Therefore, novel approaches to induce effective osteoinduction are required.

CORMs are a novel group of compounds that are carriers of $\mathrm{CO}$ and reproduce its biological actions (18). The anti-inflammatory properties of CORMs have previously been identified in vitro and in vivo $(15,20)$. It has been reported that CORM-3 inhibits the expression of adhesion molecules on human gingival fibroblasts concurrently stimulated by tumor necrosis factor- $\alpha$ and interleukin-1 $\beta$ (30). It has also been identified that the systemic administration of CORM-2 reduces periodontal inflammation and alveolar bone loss in rats with experimental periodontitis (31). To further outline the cause for reduced bone loss, it was hypothesized that CORMs may not only inhibit bone loss by suppressing inflammation, but may also directly enhance osteogenesis and/or suppress osteoclastogenesis. Subsequently, the present study aimed to investigate the effect of CORM-3 on osteogenesis.

It is well known that Runx2, which belongs to the runt-domain gene family, is a critical transcription factor for the differentiation of BMSCs and regulates a number of downstream genes associated with differentiation (32). Furthermore, Runx2 is a marker for early osteogenic differentiation (33). When the expression of Runx 2 was inhibited in fetal mice, the skeletal systems of these mice subsequently exhibited a complete lack of bone formation (34). OCN and OPN are markers of osteoblasts (35). The results of the present study indicated that pretreatment with CORM-3 significantly upregulated the expression of Runx $2, \mathrm{OCN}$ and OPN during osteogenic induction from day 3. On day 7, CORM-3 pretreatment enhanced levels of Runx 2 mRNA by 4.9 -fold compared with the control group and 2.2-fold compared with osteogenic group. These results suggest that CORM-3 may promote osteogenic differentiation. Notably, the present results also suggest that this effect may be mediated by the release of $\mathrm{CO}$.

ALP, which is considered to be an early marker of osteogenic differentiation, serves an important role in regulating cell differentiation. ALP produces inorganic phosphate from pyrophosphate (36). Phosphate is further crystallized with calcium and accelerates mineralization. The increased activity of ALP provides favorable conditions for the mineralization process and promotes the differentiation of cells into osteoblasts (37). Based on our unpublished data, the expression of heme oxygenase-1 (HO-1) mRNA and protein in BMSCs is significantly increased $24 \mathrm{~h}$ following treatment with CORM-3. Several studies have suggested that the change in HO-1 expression in response to different stimuli is consistent with that of ALP activity and demonstrated that the overexpression of HO-1 increases ALP activity $(38,39)$. In the present study, CORM-3 pretreatment significantly increased ALP activity after 3 days culture and these levels were maintained following 7 days culture. The formation of mineralized nodules is another essential marker of osteogenesis and in the current study, CORM-3 pretreatment significantly enhanced cell mineralization on day 21 .

Despite the known toxicity of $\mathrm{CO}$ at high concentrations, previous studies have revealed that low concentrations of CO may exhibit vasoregulatory properties (13) and modulate inflammation (15). A clinical study by Bathoorn et al (40) demonstrated the feasibility of administering inhaled $\mathrm{CO}$ to humans with chronic obstructive pulmonary disease. Notably, an advantage of CORMs is that they deliver CO to tissues that exhibit lower levels of carboxyhemoglobin build-up, which is typical of inhaled $\mathrm{CO}(18)$. Although CORMs have potential to be used in treatment, further pharmacokinetic and toxicological response analyses of CORMs are required prior to their clinical application.

In conclusion, the results of the present study indicate that CORM-3 promotes the osteogenic differentiation of BMSCs by releasing $\mathrm{CO}$, suggesting CORM-3 may be developed as a method of stimulating bone regeneration. However, the molecular mechanisms of its action require further elucidation. $\mathrm{CO}$ is a well-known secondary messenger involved in a range of physiological and pathological responses via interaction with specific receptors (41). The primary signaling pathways of $\mathrm{CO}$ include the HO-1, mitogen-activated protein kinase and glutathione signaling pathways $(14,15,42)$. Further studies are required to assess the mechanism by which CORM-3 promotes the osteogenic differentiation of BMSCs.

\section{Acknowledgements}

The present study was supported by Shandong Province Natural Science Foundation (grant no.ZR2015HM019), Jinan College and University Science and Technology Innovation Program (grant no. 201401259) and Special Funds for Education and Awards of Shandong Province [grant no. Lu Cai Jiao Zhi (2014) 94]. 


\section{Competing interests}

The authors declare that they have no competing interests.

\section{References}

1. Rauh J, Milan F, Günther KP and Stiehler M: Bioreactor systems for bone tissue engineering. Tissue Eng Part B, Rev 17: 263-280, 2011.

2. Loesche WJ and Grossman NS: Periodontal disease as a specific, albeit chronic,infection: Diagnosis and treatment. Clin Microbio Rev 14: 727-752, table of contents, 2001.

3. Orlic D, Kajstura J, Chimenti S, Jakoniuk I, Anderson SM, Li B, Pickel J, McKay R, Nadal-Ginard B, Bodine DM, et al: Bone marrow cells regenerate infarcted myocardium. Nature 410 701-705, 2001

4. Pittenger MF, Mackay AM, Beck SC, Jaiswal RK, Douglas R, Mosca JD, Moorman MA, Simonetti DW, Craig S and Marshak DR: Multilineage potential of adult human mesenchymal stem cells. Science 284: 143-147, 1999.

5. In't Anker PS, Scherjon SA, Kleijburg-van der Keur C, de Groot-Swings GM, Claas FH, Fibbe WE and Kanhai HH: Isolation of mesenchymal stem cells of fetal or maternal origin from human placenta. Stem Cells 22: 1338-1345, 2004.

6. Murphy MB, Moncivais K and Caplan AI: Mesenchymal stem cells: Environmentally responsive therapeutics for regenerative medicine. Exp Mol Med 45: e54, 2013.

7. Aubin JE: Regulation of osteoblast formation and function. Rev Endoc Metab Disord 2: 81-94, 2001.

8. Long F: Building strong bones: Molecular regulation of the osteoblast lineage. Nat Rev Mol Cell Biol 13: 27-38, 2011.

9. Zomorodian E and Baghaban Eslaminejad M: Mesenchymal stem cells as a potent cell source for bone regeneration. Stem Cells Int 2012: 980353, 2012.

10. Jones E and Yang X: Mesenchymal stem cells and bone regeneration: Current status. Injury 42: 562-568, 2011

11. Xiao Y, Mareddy S and Crawford R: Clonal characterization of bone marrow derived stem cells and their application for bone regeneration. Int J Oral Sci 2: 127-135, 2010.

12. Motterlini R, Gonzales A, Foresti R, Clark JE, Green CJ and Winslow RM: Heme oxygenase-1-derived carbon monoxide contributes to the suppression of acute hypertensive responses in vivo. Circ Res 83: 568-577, 1998

13. Sammut IA, Foresti R, Clark JE, Exon DJ, Vesely MJ, Sarathchandra P, Green CJ and Motterlini R: Carbon monoxide is a major contributor to the regulation of vascular tone in aortas expressing high levels of haeme oxygenase-1. Br J Pharmacol 125 : 1437-1444, 1998

14. Zhang X, Shan P, Otterbein LE, Alam J, Flavell RA, Davis RJ, Choi AM and Lee PJ: Carbon monoxide inhibition of apoptosis during ischemia-reperfusion lung injury is dependent on the p38 mitogen-activated protein kinase pathway and involves caspase 3. J Biol Chem 278: 1248-1258, 2003.

15. Otterbein LE, Bach FH, Alam J, Soares M, Tao Lu H, Wysk M, Davis RJ, Flavell RA and Choi AM: Carbon monoxide has anti-inflammatory effects involving the mitogen-activated protein kinase pathway. Nat Med 6: 422-428, 2000.

16. Matsumoto M, Makino Y, Tanaka T, Tanaka H, Ishizaka N, Noiri E, Fujita T and Nangaku M: Induction of renoprotective gene expression by cobalt ameliorates ischemic injury of the kidney in rats. J Am Soc Nephrol 14: 1825-1832, 2003.

17. Sikorski EM, Hock T, Hill-Kapturczak N and Agarwal A: The story so far: Molecular regulation of the heme oxygenase-1 gene in renal injury. Am J Physiol Renal Physiol 286: F425-F441, 2004

18. Motterlini R, Mann BE, Johnson TR, Clark JE, Foresti R and Green CJ: Bioactivity and pharmacological actions of carbon monoxide-releasing molecules. Curr Pharm Des 9: 2525-2539, 2003.

19. Foresti R, Hammad J, Clark JE, Johnson TR, Mann BE, Friebe A, Green CJ and Motterlini R: Vasoactive properties of CORM-3, a novel water-soluble carbon monoxide-releasing molecule. Br J Pharmacol 142: 453-460, 2004.

20. Jiang L, Fei D, Gong R, Yang W, Yu W, Pan S, Zhao M and Zhao M: CORM-2 inhibits TXNIP/NLRP3 inflammasome pathway in LPS-induced acute lung injury. Inflamm Res 65 905-915, 2016.

21. Livak KJ and Schmittgen TD: Analysis of relative gene expression data using real-time quantitative PCR and the $2^{-\Delta \Delta C_{\mathrm{T}}}$ method. Methods 25: 402-408, 2001.
22. Seo BM, Miura M, Gronthos S, Bartold PM, Batouli S, Brahim J, Young M, Robey PG, Wang CY and Shi S: Investigation of multipotent postnatal stem cells from human periodontal ligament. Lancet 364: 149-155, 2004

23. Tsai TL and Li WJ: Identification of bone marrow-derived soluble factors regulating human mesenchymal stem cells for bone regeneration. Stem cell Rep 8: 387-400, 2017.

24. Vahabi S, Torshabi M and Mohammadi M: Osteoinductive activity of DFDBA materials versus growth factors on gene expression of MG-63 cells: An in vitro study. J Long Term Eff Med Implants 26: 133-142, 2016.

25. Gothard D, Smith EL, Kanczler JM, Rashidi H, Qutachi O, Henstock J, Rotherham M, El Haj A, Shakesheff KM and Oreffo RO: Tissue engineered bone using select growth factors: A comprehensive review of animal studies and clinical translation studies in man. Eur Cells Mater 28: 166-208, 2014.

26. Bessa PC, Casal M and Reis RL: Bone morphogenetic proteins in tissue engineering: The road from the laboratory to the clinic, part I (basic concepts). J Tissue Eng Regen Med 2: 1-13, 2008.

27. Bessa PC, Casal M and Reis RL: Bone morphogenetic proteins in tissue engineering: The road from laboratory to clinic, part II (BMP delivery). J of Tissue Eng Regen Med 2: 81-96, 2008.

28. Fu R, Selph S, McDonagh M, Peterson K, Tiwari A, Chou R and Helfand M: Effectiveness and harms of recombinant human bone morphogenetic protein-2 in spine fusion: A systematic review and meta-analysis. Ann Intern Med 158: 890-902, 2013.

29. Mesfin A, Buchowski JM, Zebala LP, Bakhsh WR, Aronson AB, Fogelson JL, Hershman S, Kim HJ, Ahmad A and Bridwell KH: High-dose rhBMP-2 for adults: Major and minor complications: A study of 502 spine cases. J Bone Joint Surg Am 95: 1546-1553, 2013.

30. Zhao HQ, Hou M, Wei LL, Mu P, Song H and Yang P: Mechanism of carbon monoxide affecting the expression of cellular adhesion molecule under stimulation of inflammatory cytokines to human gingival fibroblasts. Hua Xi Kou Qiang Yi Xue Za Zhi 31: 420-424, 2013 (In Chinese).

31. Wei L, Hou M, Wang P and Song H: Effect of carbon monoxide releasing molecule on experimental periodontitis in rats. Hua Xi Kou Qiang Yi Xue Za Zhi 32: 23-26, 2014 (In Chinese).

32. Deng Y, Wu S, Zhou H, Bi X, Wang Y, Hu Y, Gu P and Fan X: Effects of a miR-31, Runx2, and Satb2 regulatory loop on the osteogenic differentiation of bone mesenchymal stem cells. Stem Cells Dev 22: 2278-2286, 2013.

33. Schroeder TM, Jensen ED and Westendorf JJ: Runx2: A master organizer of gene transcription in developing and maturing osteoblasts. Birth Defects Res C Embryo Today 75: 213-215, 2005.

34. Komori T, Yagi H, Nomura S, Yamaguchi A, Sasaki K, Deguchi K, Shimizu Y, Bronson RT, Gao YH, Inada M, et al: Targeted disruption of $C b f a l$ results in a complete lack of bone formation owing to maturational arrest of osteoblasts. Cell 89: 755-764, 1997.

35. Ching HS, Luddin N, Rahman IA and Ponnuraj KT: Expression of odontogenic and osteogenic markers in DPSCs and SHED: A review. Curr Stem Cell Res Ther 12:71-79, 2017.

36. Terkeltaub RA: Inorganic pyrophosphate generation and disposition in pathophysiology. Am J Physiol Cell Physiol 281: C1-C11, 2001

37. Wennberg C, Hessle L, Lundberg P, Mauro S, Narisawa S, Lerner UH and Millán JL: Functional characterization of osteoblasts and osteoclasts from alkaline phosphatase knockout mice. J Bone Miner Res 15: 1879-1888, 2000.

38. Vanella L, Kim DH, Asprinio D, Peterson SJ, Barbagallo I, Vanella A, Goldstein D, Ikehara S, Kappas A and Abraham NG: HO-1 expression increases mesenchymal stem cell-derived osteoblasts but decreases adipocyte lineage. Bone 46: 236-243, 2010.

39. Barbagallo I, Vanella A, Peterson SJ, Kim DH, Tibullo D, Giallongo C, Vanella L, Parrinello N, Palumbo GA, Di Raimondo F, et al: Overexpression of heme oxygenase-1 increases human osteoblast stem cell differentiation. J Bone Miner Metab 28: 276-288, 2010.

40. Bathoorn E, Slebos DJ, Postma DS, Koeter GH, van Oosterhout AJ, van der Toorn M, Boezen HM and Kerstjens HA: Anti-inflammatory effects of inhaled carbon monoxide in patients with COPD: A pilot study. Eur Respir J 30: 1131-1137, 2007.

41. Levitt DG and Levitt MD: Carbon monoxide: A critical quantitative analysis and review of the extent and limitations of its second messenger function. Clin Pharmacol 7: 37-56, 2015.

42. Otterbein LE, Foresti R and Motterlini R: Heme oxygenase-1 and carbon monoxide in the heart: The balancing act between danger signaling and pro-survival. Circ Res 118:1940-1959, 2016 